\title{
In-situ Transmission Electron Microscopy of Biological Specimens in Liquid
}

\author{
Madeline J. Dukes $^{1}$, Sarah M. McDonald ${ }^{2}$ and Deborah F. Kelly ${ }^{2}$ \\ 1. Applications Science, Protochips, Inc., Raleigh, NC, USA \\ 2. Virginia Tech Carilion Research Institute, 2 Riverside Circle, Roanoke, VA, USA
}

Transmission electron microscopy (TEM) allows us to peer into the world of nanomaterials and biological processes while approaching near-atomic resolution ${ }^{1}$. Conventionally, in order to take advantage of resolution that TEM provides, specimens must be dried or immobilized in ice or plastic resin prior to being inserted into the vacuum of the microscope column. The need to observe hydrated species at high resolution has spurred the development of in-situ sample holders that isolate liquid specimens from the column vacuum by enclosing them between thin membranes of electron transparent materials $^{2-4}$.

Here we describe recent results using a commercially available liquid TEM holder to observe biological and soft materials in situ ${ }^{5}$. Within the tip of the holder, a liquid specimen is enclosed between to silicon microchips each containing a 50-nm thick silicon nitride membrane that serves as a transparent viewing window (Figure 1). Integrated microfluidic tubing enables liquid to be flowed into and through the sample chamber while imaging without disassembling the liquid cell. Thus, the liquid environment can be dynamically tuned to observe processes as they occur in real-time.

A current limitation with in situ techniques is that specimens in liquid can freely move, thus limiting the resolution at which they can be imaged. In order to facilitate the capture of biological assemblies within the microfluidic chamber, the surface of the microchips were modified with an "affinity" coating (Figure 2A). A lipid biofilm doped with functionalized Ni-NTA lipids and DLPC filler lipids, was deposited onto the surface of the microchip that formed the bottom platform of the sample chamber. As an initial proof-of-concept experiment, His-tagged ribosomes produced in bacterial cell lysates were purified and imaged in liquid. We accomplished this by flowing the lysate into the chamber lined with the Ni-NTA functionalized surface. The concentration of ribosomes (Figure 2B) increased over time until saturation of the Ni-NTA lipids was achieved ${ }^{6}$. Another biological application, presented here, utilized an affinity-coating tethered with polyclonal antibodies to capture rotavirus double layer particles (DLPs) ${ }^{7}$. The DLPs were contained in a $150 \mathrm{~nm}$ thick liquid layer along with a low concentration of contrast reagent $(0.2 \% \mathrm{w} / \mathrm{v}$ uranyl formate) to enhance downstream image processing (Figure $2 \mathrm{C}$ ). An analogous dataset of rotavirus DLPs was also obtained using cryo-TEM, and 3D reconstructions were calculated using the RELION software package (Figure 2C, inset). Resolutions of $25 \AA$ and $24 \AA$ were obtained for the in-situ and cryo-TEM samples, respectively. Statistical analysis of the images revealed that the viral assemblies imaged in solution exhibited a greater degree of structural heterogeneity than those fixed in vitreous ice.

Overall, we demonstrate the use of in situ TEM as a new frontier for imaging biological specimens in a liquid, native environment. We have developed affinity capture strategies that enable the isolation of biological complexes for in situ liquid TEM imaging, which mitigated diffusion of the complexes in solution. This provides, for the first time, a useful strategy to produce specimens in solution that are suitable for single particle image processing routines and 3D reconstruction calculations. This novel application may bridge the gap between cellular and molecular imaging techniques. 


\section{References:}

(1) Leis, A.; Rockel, B.; Andrees, L.; Baumeister, W. Trends Biochem.Sci. 2009, 34, 60-70.

(2) Yuk, J. M.; Park, J.; Ercius, P.; Kim, K.; Hellebusch, D. J.; Crommie, M. F.; Lee, J. Y.; Zettl, A.; Alivisatos, A. P. Science 2012, 336, 61-64.

(3) Klein, K. L.; Anderson, I. M.; De Jonge, N. Journal of Microscopy 2011, no-no.

(4) Dukes, M. J.; Peckys, D. B.; de Jonge, N. Acs Nano 2010, 4, 4110-4116.

(5) Ring, E. A.; Peckys, D. B.; Dukes, M. J.; Baudoin, J. P.; De Jonge, N. Journal of Microscopy 2011, 243, 273-283.

(6) Degen, K.; Dukes, M.; Tanner, J. R.; Kelly, D. F. RSC Advances 2012, 2, 2408-2412.

(7) Gilmore, B. L.; Showalter, S. P.; Dukes, M. J.; Tanner, J. R.; Demmert, A. C.; McDonald, S. M.; Kelly, D. F. Lab on a Chip 2013, 13, 216-219.
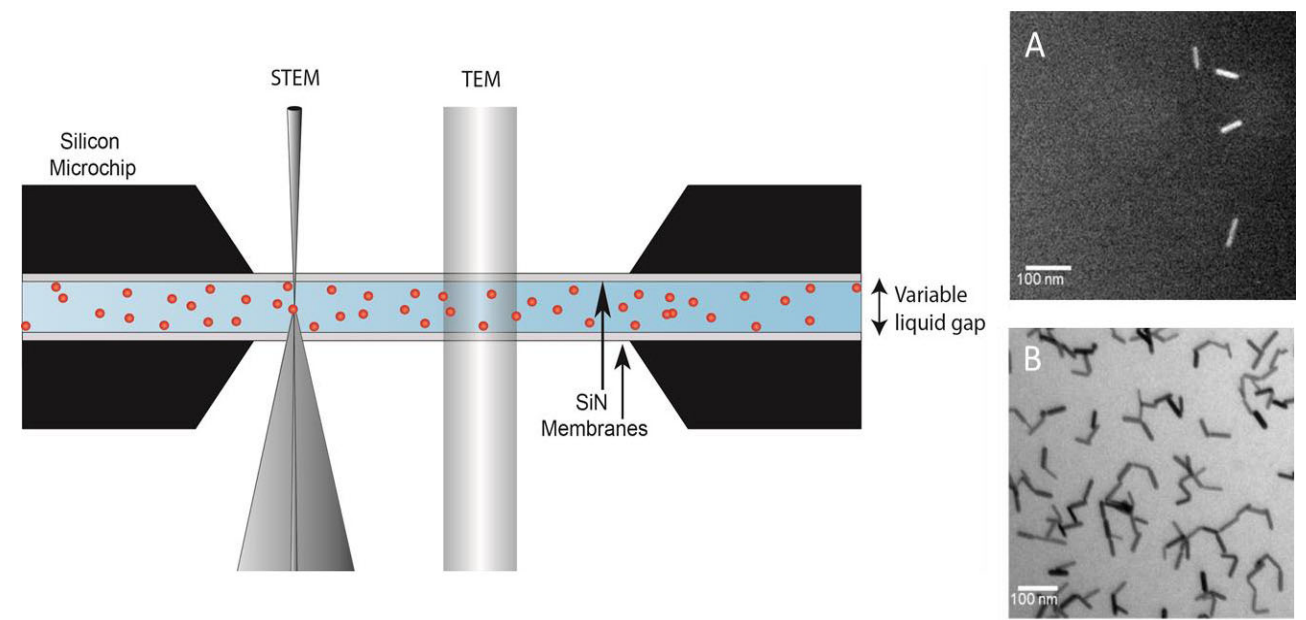

Figure 1. Cross section of liquid contained between two electron transparent, silicon nitride windows. Inset A: STEM image of gold nanorods imaged in liquid (JEOL 2100, $200 \mathrm{KV}$ ). Inset B: TEM image of gold nanorods imaged in liquid (FEI Tecnai, 120KV).
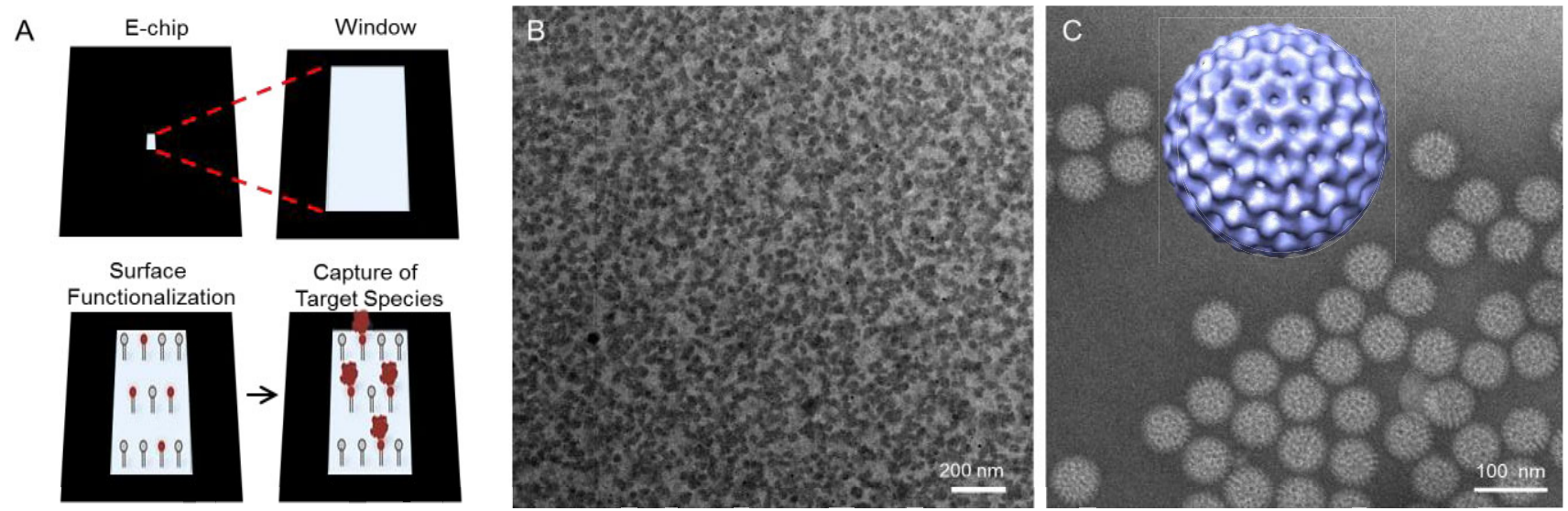

Figure 2. Imaging biological specimens using in-situ TEM. (A) Schematic showing affinity capture functionalization of silicon nitride E-chips used to produce the liquid imaging chamber. (B) Ribosomes captured in situ from bacterial lysate and imaged in $150 \mathrm{~nm}$ liquid using TEM. (C) Rotavirus double layer particles imaged in $150 \mathrm{~nm}$ liquid using TEM and corresponding 3D reconstruction (purple inset). 